\title{
Mobile Telephone
}

National Cancer Institute

\section{Source}

National Cancer Institute. Mobile Telephone. NCI Thesaurus. Code C60786.

Mobile communications system that uses a combination of radio wave transmission and conventional telephone switching to permit telephone communication to and from mobile users within a specified area. 